\title{
Findings of a Breast Assessment Service and Implications for Clothing
}

\author{
Graham HUTTON, Samantha TEMA, Martin BAYLEY \\ Body Aspect Ltd, Nottingham, UK \\ http://dx.doi.org/10.15221/13.392
}

\begin{abstract}
Since 2003, Body Aspect has collected 3D data on over 2000 female subjects as part of a breast assessment service for the UK's National Health Service (NHS). The data includes women who have sought treatment for breast reduction, asymmetry correction and augmentation. Our service is aimed at helping the NHS to decide who should be considered for those treatments. But the criteria for acceptance are stringent. For example, $70 \%$ of the women referred for consideration for breast reduction are not eligible to receive an operation. For these women, finding suitable clothing, especially a bra or swimsuit, can be more of a problem than for the majority of women. This paper presents some statistics on the data collected, discusses the issues relevant to these women and attempts to highlight potential opportunities for clothing manufacturers addressing this niche area.
\end{abstract}

\section{Introduction}

\section{About Body Aspect}

Body Aspect was established in 2003. The core of the team previously formed part of a commercial research group at Nottingham Trent University (NTU) at a time in the 1990's when NTU's Computer Clothing Research team were pioneering the modern era of sizing surveys and body shape analysis for apparel [1] using a Telmat 2D image capture system.

Since its formation, Body Aspect has worked with plastic surgeons and NHS commissioning teams to develop a new pathway for elective breast reduction mammaplasty; a pathway that is objective and equitable. The company has focused on this niche area of activity, whilst maintaining an interest in related applications and projects within the healthcare and apparel fields.

One other interesting activity has been the development of a prototype Body Image assessment tool which can allow subjects to compare their perceived image against their actual image. This has been featured in the media [2, 3]. A variant of this software was developed as a prototype to help motivate weight management.

\section{Background to Breast Assessment Service}

In the early 2000's, NHS commissioners, surgeons and patients in the Trent region were affected by the lack of a clear and equitable pathway for breast reduction. This stemmed from a general lack of agreement on how to decide who should be eligible for Breast Reduction within the NHS. This led to a growing waiting list, inconsistency, poor use of resources and ultimately patient dissatisfaction.

As a result of our background in 3D body scanning for lingerie, our team were consulted to help solve the problem. A trial was conducted and criteria were agreed that took into account breast volume and the relative size of the breasts in relation to the thoracic region. This data is captured using 3D body scanning. The resulting service allows each patient to be assessed against the same objective criteria with a report being provided to the commissioning organisation [4].

From the initial work, it became clear that the 3D data could also be used to assess breast asymmetry. As a result, the remit of the service was extended to incorporate these patients too. A small number of commissioning groups also use the Body Aspect service to assess breast augmentation patients. In such cases, the criteria often stipulates that the patient should have a complete absence of breast tissue. In practice this is hard to assess, so body scanning can provide objective data on which a decision can be made.

In terms of patient numbers, it is interesting to observe that although the service only involves scanning a trickle of patients (approximately four or five per week), over time these can accumulate to form a sizeable sample. During the period July 2003 to July 2013 a total of 2252 subjects were scanned, comprised of 1575 assessed for breast reduction, 555 for asymmetry and 122 for augmentation. 


\section{Problems affecting women with large or different-sized breasts}

It is normal for breast size and breast shape to vary between women. However, in some cases where a woman has particularly small or large breasts, or where differences in breast size or shape are pronounced, it can cause problems.

\section{Disproportionately large breasts}

For most women, breast size is proportionate to the body, but for some, the breasts are particularly large. This may incur a number of problems [5], including:

- poor posture

- weals or grooves on the shoulders from bra straps

- not being able to wear fashionable clothes

- backache, neck pain, skin irritation, excessive sweating, rashes and skin infections under the breasts

- an inability to exercise or take part in sports

- unwanted attention or harassment

- self-consciousness

- negative effects on mental and emotional wellbeing

\section{Breast asymmetry}

Many women have one breast which is slightly larger than the other, but when this is pronounced it can lead to the woman becoming particularly self-conscious about her appearance. Some problems are as follows:

- finding a bra that will be suitable for breasts of different cup size.

- having to wear padding in the bra

- being self conscious about appearance, leading to depression, or relationship difficulties

- avoidance of wearing certain types of clothes

- avoidance of swimming due to unavailability of costume that would accommodate suitable prosthesis

- resorting to means of masking difference e.g. tattooing one side

\section{Methods}

\section{Use of 3D Body Scanning within the Breast Assessment Service}

We use a 3D body scanner to capture a full body image. The full body data is useful for us to compare breast size in relation to the torso. Our requirements for a system are that it should be totally safe, accurate to acceptable tolerances and dignified for the patients. We export the raw data into our software which allows us to create a structured but anonymous representation of the body [6]. From this we can calculate breast volume and torso volume.

\section{Body scanning hardware}

For ten years we used a Wicks and Wilson Triform scanner [website no longer available]. This system used structured white light to capture an image in about 12 seconds. The system worked reliably provided we did regular maintenance which included: a quarterly calibration; replacement of interior bulbs; cleaning of mirrors; and annual replacement of module bulbs and gauze filters. Owing to its use of white light, the system was poor at detecting hair and black clothing, but these were not particular problems for our application. However, it occasionally had problems with subjects who had a black, shiny skin tone. This could be tackled by adjusting settings in the scanner's software, but it made the capture process more complicated.

In May 2013, we replaced the system with a SizeStream 3D body scanner [7]. This system uses infrared sensors and has proven to show no issues relating to dark clothes, hair or skin. The configuration of fourteen sensors gives a good coverage of the body, and the overall footprint is smaller than the previous scanner. The footprint may be a consideration for some users, but for our applications a greater priority is to ensure that we can scan subjects of all sizes. The capture time is also less than our previous system, which is important in reducing subject movement. One advantage of the previous system was that it had fixed handholds which locked into place; these 
helped a subject to maintain a stable pose. Calibration needs to be performed frequently (daily) but is a simple process. At the time of writing the system has only been operational for three months, so it is not possible for us to comment on the long-term durability of the system. The system comes with software for automatically processing a scan and extracting measurements. The assessment process imports the raw data into our bespoke software which allows specialised cleaning and landmark selection to generate a tailored chest wall model for each individual patient.

\section{Body Aspect Software}

As mentioned previously, the SizeStream system comes with an automated processing capability. But it is still hard for any system to reliably process a scan $100 \%$ of the time. For this reason we take the raw data and process it in our own semi-automated software. In particular the following tasks are performed with some expert user supervision:

- removing spurious data

- positioning of key landmarks for body segmentation

- identifying data for chest wall interpolation

We have found that accuracy and reliability are improved by being able to manually refine the chest wall model-fitting process to take account of scan quality issues and unusual body morphologies. As such, our tools to enable this process have been constantly evolving.

We compute breast volume by examining the difference between the surface data and the interpolated chest wall. The software and methods for calculating the volumetric data were scrutinised by the East Midlands Clinical Priorities Advisory Group. Internal testing, using simulated breasts of different volumes, led to an estimated mean accuracy of less than $5 \%$ when calculating breast volume. This is a considerable improvement on the ability of a surgeon to estimate the volume 'by eye' [8].

In order to assess the proportionate breast size for breast reduction and augmentation patients, we also compute a measure of upper body torso volume (with breast volume subtracted) which is normalised to take account of torso length. This offers an objective measure of the proportional size of the breasts, and is regarded as considerably better than the more subjective criteria that had been used previously [8].

The software also has the ability to interrogate a database of scans in order to filter according to criteria such as breast volume, girth measurements, BMI or bra size. Through use of a landmarkframed canonical model definition [6] we are easily able to create a meaningful 'average' 3D model for any subset of body models.

\section{Additional manual measurements}

For the purpose of the breast assessment service we supplement the scan data with some standard additional manual measurements:

- bust girth

- underbust girth

- waist girth

- hip girth.

These are not used as part of the assessment but are retained as a practical means of integrity checking in the case of scan anomalies.

To assist in the assessment of breast shape asymmetry, the following measurements are also collected:

- sternal notch to nipple

- nipple to infra-mammary fold

- areola diameter

Although it is possible to estimate these measurements from the scan data, which would be preferable in terms of patient dignity, we believe the accuracy and credibility of the data to the patient is improved by a nurse operator taking these measurements manually. 


\section{Considerations when scanning larger bust sizes}

When scanning for clothing applications it is quite common for subjects to be scanned in a standing pose with arms slightly away from the side of the body. Such a pose enables most of the body to be captured whilst maintaining a natural position that facilitates body measurement extraction. However, in order to capture good data around the breast region for the purpose of accurately estimating breast volume, the scan data needs to capture data at the side of the breasts. For larger bust sizes this means that the subject must raise the arms higher than the common scan position.

One issue has been to decide whether to scan subjects with or without a bra. With larger breast volumes, e.g. those over $1000 \mathrm{cc}$, the breast can spread out over a larger area and becomes difficult to distinguish from the chest wall. For this reason, breast reduction scans are taken wearing a bra. This is a trade-off, because if the bra is poorly-fitting then there may be an additional error introduced. We can minimise the effect of poorly-fitted bras by fitting the patient prior to the scan. The current consensus, based on experimentation and experience, is that both accuracy and patient experience is improved when the subject is wearing a bra. For asymmetry scans, wearing a bra is not an option, and in cases where a patient is considered for both asymmetry and reduction, scans are captured both with and without a bra.

\section{Results}

The following data presents a summary of data collected during the last ten years.

\section{Distribution of breast volume for patients seeking breast reduction}

Figure 1 and Table 1 show the distribution of breast volumes for patients seeking breast reduction treatment. The mean volume for patients seeking treatment is $1344 \mathrm{cc}$, whereas the mean volume for patients passing the assessment criteria is $1604 \mathrm{cc}$. In terms of comparing this with the general UK population, the mean volume for those patients who typically wear a $36 \mathrm{D}$ bra was $750 \mathrm{cc}$.

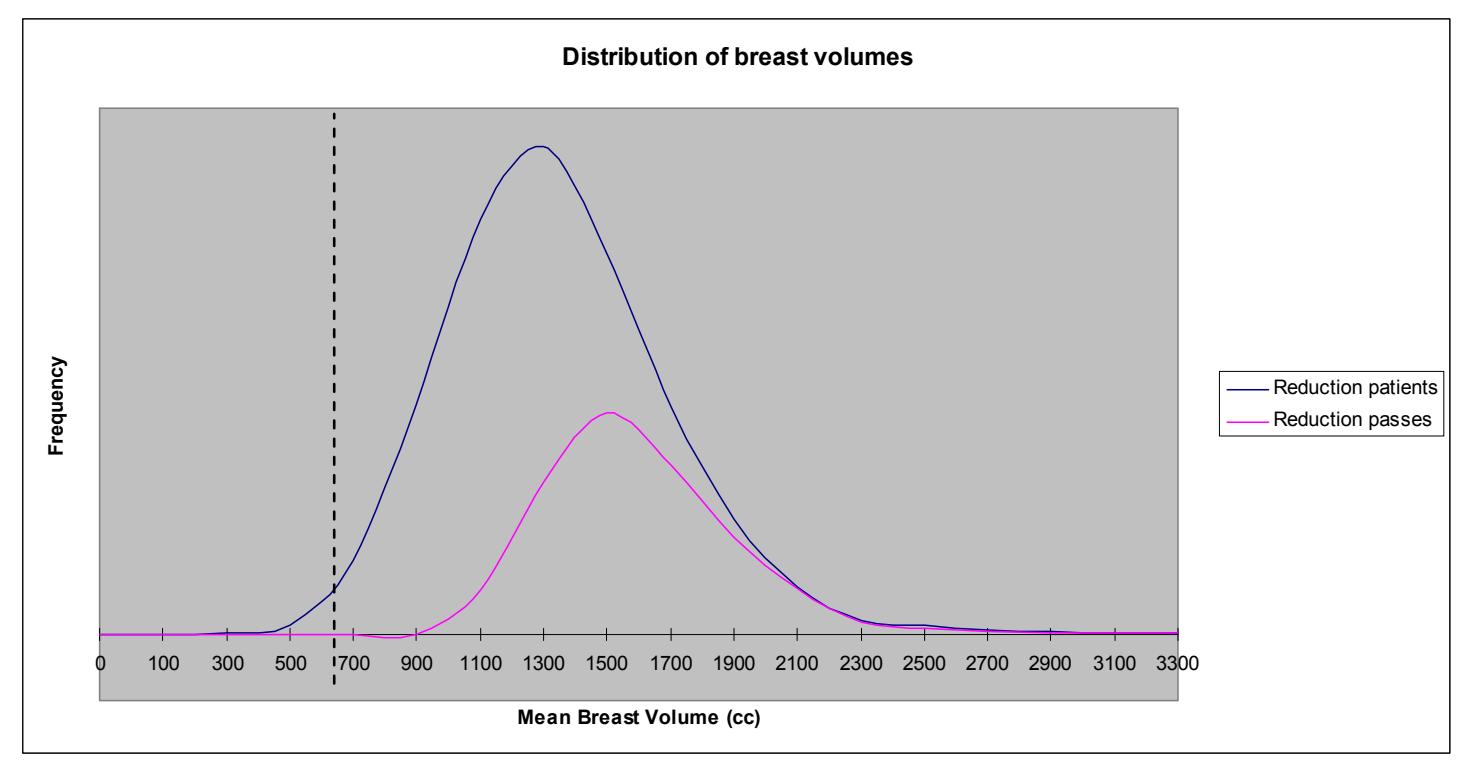

Fig. 1. Graph of Distribution of breast volumes for (a) reduction patients $(n=1540)$ and (b) those who pass the criteria ( $n=578)$. The dashed line shows the mean volume for patients who typically wear a 34D bra.

Table 1. Summary statistics for breast volume of breast reduction patients

\begin{tabular}{|c|c|c|}
\hline & Reduction patients & Reduction Passes \\
\hline $\mathrm{N}$ & 1540 & 578 \\
\hline Mean volume (cc) & 1344 & 1604 \\
\hline Standard Deviation & 358 & 312 \\
\hline
\end{tabular}




\section{Distribution of volumetric difference for subjects seeking asymmetry correction}

Many women have one breast that is slightly larger than the other. Previous sizing survey experience means that we can estimate that within the UK population the average asymmetry, in terms of breast volume difference, is approximately $10 \%$, and that there is a large variation in the degree of asymmetry. Figure 2 and Table 2 show the distribution of volumetric difference for patients being assessed for asymmetry correction, where the mean difference in breast volume is $27.2 \%$ (expressed as a percentage of the larger breast). In absolute terms the mean difference is $161 \mathrm{cc}$ which equates to one or two cup sizes. However, in more extreme cases, patients can have a percentage difference greater than $60 \%$ or an absolute difference of $700 \mathrm{cc}$, which is enough to span approximately five cup sizes (figure 3).

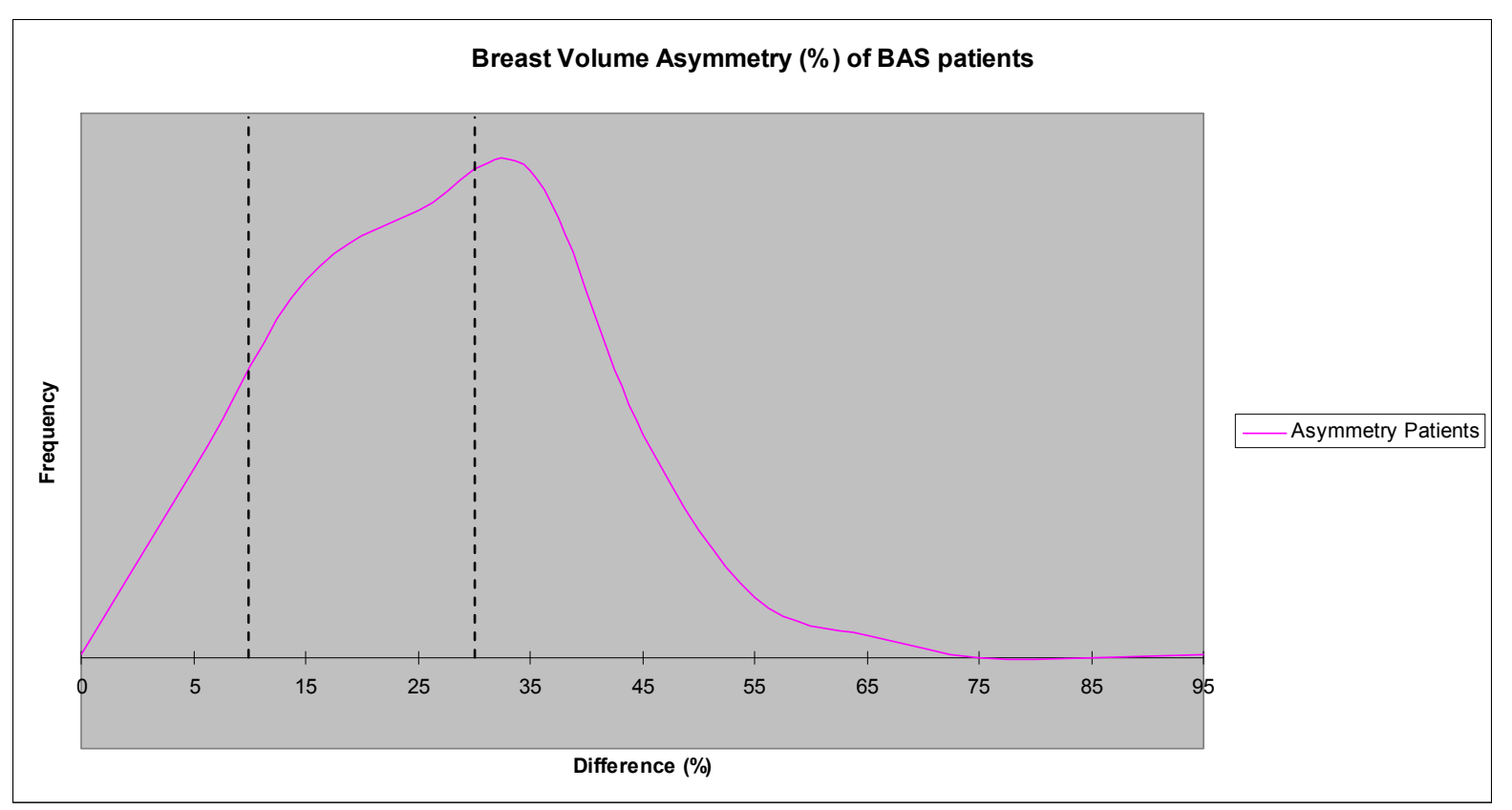

Fig. 2. Distribution of breast volume differences for asymmetry patients ( $n=563)$. Estimate of population mean asymmetry (10\%) and the common threshold for acceptance for treatment (30\%) are indicated by dashed lines.

Table 2. Summary statistics for breast volume difference of breast asymmetry patients

\begin{tabular}{|c|c|c|}
\hline & $\begin{array}{c}\text { Difference as \% of } \\
\text { larger breast }\end{array}$ & $\begin{array}{c}\text { Absolute difference } \\
\text { (cc) }\end{array}$ \\
\hline Mean & 27.2 & 161 \\
\hline Standard Deviation & 13.6 & 115 \\
\hline
\end{tabular}

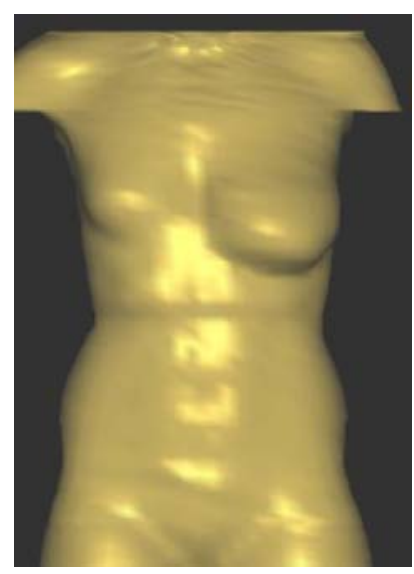

Figure 3. Example of breast asymmetry (60\% difference) 


\section{Perceived bra size against breast volume of breast reduction patients}

For each patient we recorded the bra size that they typically wear. Table 3 shows the mean volume corresponding to each cup size. It should be borne in mind that although these subjects were advised to have a professional bra fitting prior to their scan, there were still some with poorly fitting bras.

Table 3. Perceived bra size $v$ mean breast volume (each cell is the mean of at least 10 data points)

\begin{tabular}{|c|c|c|c|c|c|c|c|c|c|c|c|}
\hline UK & D & DD & E & F & FF & G & GG & H & HH & J & JJ \\
\hline US & $D$ & $D D$ & $D D D$ & $G$ & $H$ & $I$ & $J$ & $K$ & $L$ & $M$ & $N$ \\
\hline $\mathbf{3 0}$ & & & & & 884 & 1035 & 1143 & 1298 & 1331 & 1594 & 1595 \\
\hline $\mathbf{3 2}$ & & & 794 & 955 & 1043 & 1122 & 1284 & 1388 & 1559 & 1641 & 1683 \\
\hline $\mathbf{3 4}$ & & 829 & 996 & 1114 & 1239 & 1308 & 1468 & 1556 & 1728 & 1748 & 1949 \\
\hline $\mathbf{3 6}$ & 754 & 941 & 1180 & 1230 & 1290 & 1464 & 1604 & 1769 & 1557 & 2004 & \\
\hline $\mathbf{3 8}$ & & 1129 & 1251 & 1304 & 1420 & 1578 & & & & 1955 & \\
\hline $\mathbf{4 0}$ & & & & 1635 & & & & & & & \\
\hline
\end{tabular}

\section{Variation in the breast volume of women who wear a 34G bra}

The most common bra size amongst those women seeking breast reduction patients is 34G. Figure 4 shows the distribution of volumes for those women, who all wear the same size bra. The mean breast volume is $1308 \mathrm{cc}$ but there is a great variation ranging from $670 \mathrm{cc}$ to $2306 \mathrm{cc}$ (standard deviation $=248$ ). One obvious inference from this is that perceived bra size is not a good indicator of breast volume.

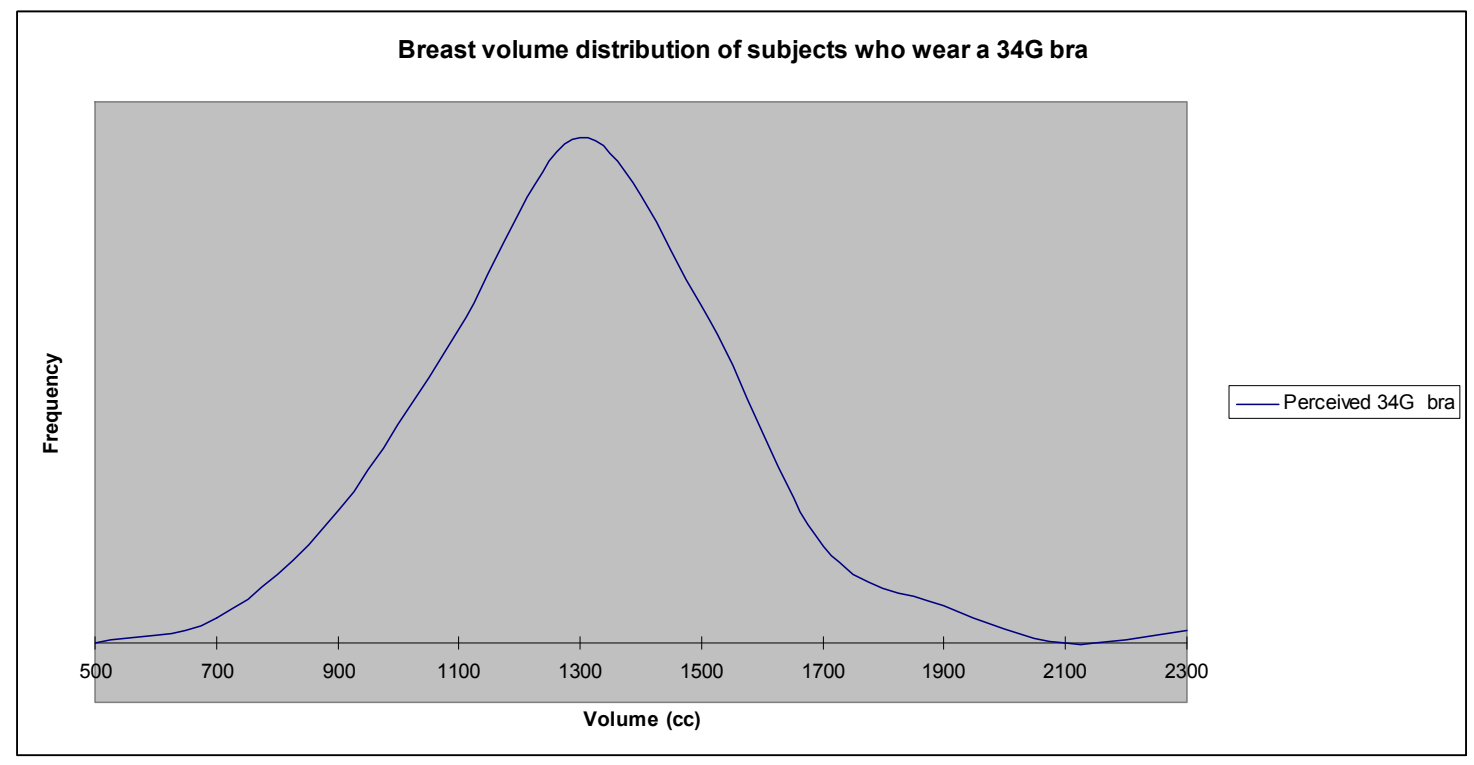

Figure 4. Distribution of breast volume for patients wearing a $34 G$ bra $(n=121)$

\section{Effect of large breasts on body shape and posture}

In terms of body shape, Figure 5 shows two 3D-averaged body model images. The left image is the average of ten women with small breast volume $(0-300 \mathrm{cc})$. The right image shows the average of ten women who have large breast volume $(1200-1700 \mathrm{cc})$. The subjects used to create the averaged images each had a similar Body Mass Index (within $1 \mathrm{~kg} / \mathrm{m}^{2}$ ). Although the bust differs noticeably, there does not appear to be much difference in the shape of the waist and hips. One inference from this could be that the shape of the bust occurs independently of the shape of the waist and hips. 
Figure 6 shows the side view of the two averaged images, with the breasts removed. In this case it appears that the right hand image is more arched and the seat prominence is greater. Two possibilities here are (i) that posture is affected by breast size, and/or (ii) seat prominence is correlated with breast volume. These observations have not been investigated further at present. Another caveat to consider is that the posture during scanning is not the subject's normal relaxed standing position, and the effect of holding onto the hand bars with arms slightly raised, needs also to be taken into account.

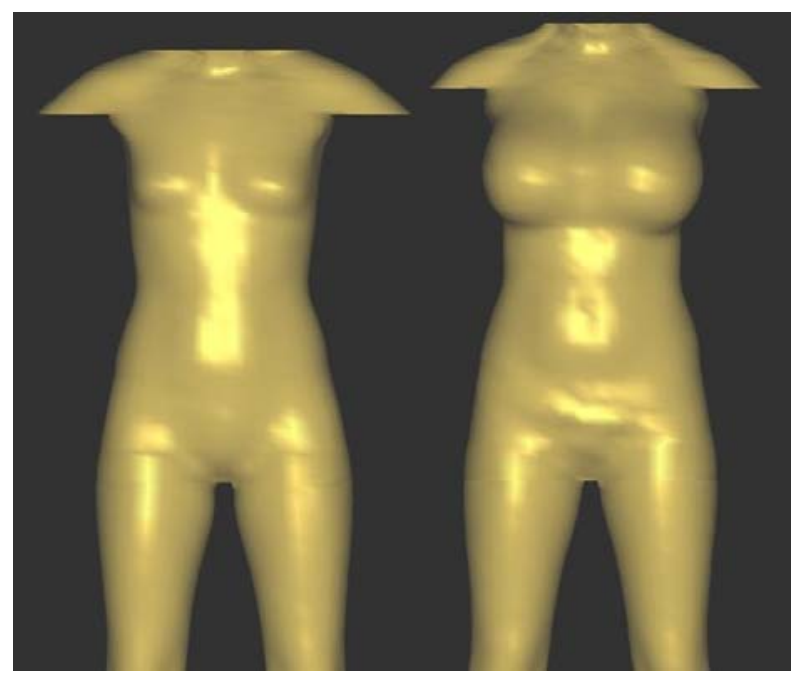

Figure 5. 3D Average of ten small breast volumes $v$ Average of ten large breast volumes (front view)

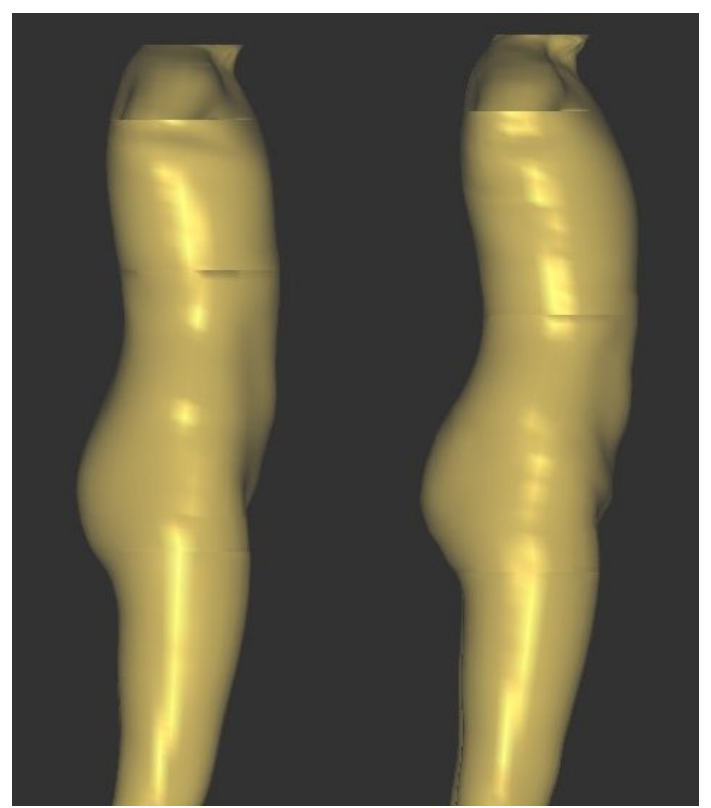

Figure 6. 3D Average of ten small breast volumes $v$ Average of ten large breast volumes (side view with breasts removed)

\section{Discussion}

This work has focused on two specific groups of women: those with disproportionately large breasts; and those with breasts that are significantly different in size. Currently, in the UK, the most severe cases will qualify for surgery to alleviate the problem. However, many will not qualify, or will choose not to have surgery. For these women the issue of finding a suitable bra, garment or swimming costume can be a significant problem. 


\section{Clothing for larger bust sizes}

In general there is some acknowledgement that breast size is increasing in the UK and the USA [9, 10]. It is less clear whether breasts are becoming proportionately larger or whether it is simply as a result of the population getting bigger (since there is also an acknowledgement that obesity is a growing problem). In either case this means that manufacturers are having to accommodate a greater range of breast sizes. Retailers are offering larger bra sizes such as $L$ and $N$ cups, and the market is seeing more examples of retailers focusing on clothes for these women [11]. The author predicts that this trend will continue and that more retailers will perceive the growing market for garments that accommodate this particular subset of the female population.

There is also an increasing opportunity to offer solutions that are tailored to the individual, and $3 \mathrm{D}$ scanning could provide the data to allow classification of bespoke morphology. This could directly feed into the established tailoring process by informing the modification or morphing of a standard pattern to account for personalised shape issues. It is not a trivial matter to adapt clothing patterns to suit particular individuals, as is demonstrated by the slow progress in this respect since early attempts at addressing the issue [1], but nor is it an impossible task and it is encouraging to see more activity in this area $[12,13]$.

3D Body Scanning is sometimes seen as a rapid and automated substitute to the methods of the traditional tailor but, the author believes, it should instead be seen as an additional tool that the skilled tailor can use alongside the tape measure to help understand the particular $3 \mathrm{D}$ issues that need to be addressed [14].

\section{Clothing for breast asymmetry}

Breast asymmetry is common within the female population. In cases where the asymmetry is significant, women have to choose whether to wear a bra that fits the smaller breast, the larger breast or, as a compromise, somewhere in between. This means that either one breast will not fit in the cup, or the other will be less supported, or, to a lesser degree, both. In our group of patients the asymmetry can be quite severe with left-right differences of up to five cup sizes. In many cases the problem is dealt with by padding the smaller cup.

An online search reveals that it is also possible to buy a bra that has cups of different sizes [15]. This addresses the fit and comfort aspects. But the appearance aspect will still be an issue. An alternative is to wear a pre-padded bra, with removable padding or silicone pads. And for those women with a substantial difference in breast size a potential solution is available through the use of a pocketed bra (or mastectomy bra) which accommodates a prosthesis.

In the case of asymmetry, 3D body scanning improves the scope for offering a customised solution tailored for an individual. A 3D scan of the breast region provides the means of accurate volume and shape measurement to quantify the difference between the breasts. This information could be used in the design of bespoke padding or prosthesis to restore symmetry, specifically informing the volume and footprint of the solution.

\section{Helping our patients}

Body Aspect welcomes any developments that help women to deal with the problems of disproportionately large breasts or significant breast asymmetry. Such developments can augment the service we offer by providing assistance to the majority of patients, who do not qualify for treatment on the NHS, and to those who would prefer not to have an operation. The cost of operations in the private sector is often prohibitive for those seeking treatment. But the availability of well-fitting bras and clothing can alleviate some of the problems these women endure, and we are always pleased to inform patients about new products that might improve their quality of life. 


\section{References}

1. Conferences at the NICC, Hucknall, UK: Is Size Important (1997). Mass Customisation (1998).

2. TV Series using Body Aspect's prototype Image Assessment tool: "How To Look Good Naked" presented by Gok Wan (2006). Channel 4. "The Insider" presented by Jane Moore (2007). Channel 4. "Slim To Win" presented by Rosemary Conley (2007). ITV. "Am I Normal" presented by Tanya Byron. (2008). BBC.

3. Newspaper articles referring to the Body Assessment Tool: Azoulay, J.F., (2004): "Body Scans and Consumer Demands move into the future". AATCC. Murphy-Jack, O. (2006): "New Scanning Device Offers Hope to Body Dysmorphia Sufferers". The Big Issue. Feinmann, J. (2006): "The Tyranny of Thin". The Independent.

4. NHS Modernisation Agency (2005): "Information for Commissioners of Plastic Surgery". Action-On Plastic Surgery Good Practice Guidance.

5. NHS Choices website: www.nhs.uk/conditions/Breast-reduction/

6. Hutton, G., Bayley, M., Broadhead, M. and Knox, A. (2002): "Shape Analysis for Apparel; Applications and Methods". Human Modelling, Paris.

7. www.sizestream.com/

8. Lim, P., Rimouche, S., Henley, M. (2013): "3D scanning for assessment of patients seeking breast reduction: eliminating the postcode lottery and preserving patient dignity". BAPRAS conference presentation.

9. Fisher, A. (2010): "Why are British women's breasts getting bigger?" The Guardian.

10.ASBCI Lingerie and Swimwear Seminar. (2010): "Shaping up for Lingerie and Swimwear"

11.Bravissimo: www.bravissimo.com/about/

12.Khanna, A. (2010): Building an Interface Between Surgery and the Lingerie Industry". ASBCI Lingerie and Swimwear Seminar.

13.Tailored For You: www.tfycollections.co.uk/

14.Hutton, G., Bayley, M., Broadhead, M. (2003): "Made to Measure Clothing: Applications of 3D scanning". Human Modelling, Paris.

15.www.lindasonline.com/uneven-breasts-in-bra.html 